\title{
Modeling and simulation of vacuum evaporation process through graphical interfaces
}

\section{Adriana Martínez Gómez ${ }^{1}$, Patricia Atempa Rosiles ${ }^{2}$, Anselmo Osorio Mirón ${ }^{3}$, Miguel V. Teco Ja-come ${ }^{4}$, Luis A. Sánchez Bazán ${ }^{5}$, Joaquín Santos Luna ${ }^{6}$, Alejandra Velasco Pérez ${ }^{7}$}

1,2,3,5,7 Universidad Veracruzana, Facultad de Ciencias Químicas, México. Prolongación Oriente 6 No. 1009, Col. Rafael Alvarado, C. P. Orizaba, Veracruz, México. Tel/Fax (+52) 2727240120

${ }^{4}$ Universidad Politécnica de Huatusco, México.

${ }^{6}$ Universidad Veracruzana, Facultad de Ingeniería Mecánica Eléctrica, México.

Email: amargo.89@hotmail.com,patty_156@hotmail.com, anosorio@uv.mx,mteco@hotmail.com,mc_bazan74@hotmail.com, joasantos@uv.mx, avelasco@uv.mx

Received: November $10^{\text {th }}, 2017$.

Accepted: January $15^{\text {th }}, 2018$.

Published: March $30^{\text {th }}, 2018$.

Copyright (C2016 by authors and Institute of Technology Galileo of Amazon (ITEGAM).

This work is licensed under the Creative Commons Attribution International

License (CC BY 4.0).

http://creativecommons.org/licenses/by/4.0/

\section{cc) (i) (2)(2) Open Acten:}

\begin{abstract}
This article presents the development of a software with graphical interfaces for the simulation of the evaporation process in simple and multiple effects, as part of the construction of a virtual laboratory of unitary operations in distillation and evaporation. The design and construction of the user interfaces was carried out in the QtCreator application, later the coding of the calculation algorithms was developed in the Python programming language, due to the appropriate characteristics that it presents, in Regarding the construction of graphs and methods of solving equations. The calculations carried out in the software allow the determination of temperatures and pressures, the output flows and the corresponding enthalpies; presents in graphic form the dynamic behavior of the variables incorporated in the process model. With the developed system, it is possible to perform simulations of the evaporation process in a nonstationary state for simple and multiple effects, considering solutions of sodium hydroxide, sucrose, milk, sodium chloride and sulfuric acid.
\end{abstract}

Keywords: Dynamic evaporation, graphic interfaces, virtual laboratory, programming language.

Modelado y simulación del proceso de evaporación al vacío através de interfaces gráficas

\section{RESUMEN}

En este artículo se presenta el desarrollo de un software con interfaces gráficas para la simulación del proceso de evaporación en simple y múltiple efecto, como parte de la construcción de un laboratorio virtual de operaciones unitarias en destilación y evaporación. El diseño y construcción de las interfaces de usuario se realizó en la aplicación QtCreator, posteriormente se desarrollo la codificación de los algoritmos de cálculo en el lenguaje de programación Python, debido a las características apropiadas que presenta, en cuanto a la construcción de gráficas y métodos de solución de ecuaciones. Los cálculos que se realizan en el software permiten la determinación de temperaturas y presiones, los flujos de salida y las entalpías correspondientes; presenta en forma gráfica el comportamiento dinámico de las variables incorporadas en el modelo del proceso. Con el sistema desarrollado, es posible realizar simulaciones del proceso de evaporación en estado no estacionario para simple y múltiple efecto, considerando soluciones de hidróxido de sodio

Palabras Clave: Evaporación dinámica, interfaces gráficas, laboratorio virtual, lenguaje de programación.

\section{INTRODUCCIÓN}

La Evaporación es una operación unitaria en la cual se lleva a cabo el aumento de concentración de una solución de un líquido, que se denomina solvente y uno o varios solutos sólidos disueltos en dichos solventes. Para separar estos componentes, es necesario adicionar calor en forma de vapor saturado en un lado de una superficie de contacto para que el calor latente se transfiera por conducción a través de dicha pared y por convección en el seno del líquido[1]. 
La mayoría de los evaporadores se calientan con vapor de agua que condensa sobre tubos metálicos. El material que se evapora circula casi siempre por el interior de los tubos. Generalmente se utiliza vapor de agua a baja presión, inferior a 3 $\mathrm{kg} / \mathrm{cm} 2$, y el líquido hierve a un vacio moderado, superior a 70 $\mathrm{mm} \mathrm{Hg}$ aproximadamente. $\mathrm{Al}$ disminuir la temperatura de ebullición del líquido, aumenta la diferencia de temperatura entre el vapor condensante y el liquido que hierve como consecuencia, aumenta la velocidad de transmisión de calor en el evaporador. Cuando se utiliza un solo evaporador, el vapor procedente de la ebullición del liquido se condensa y se desprecia. Este método se denomina evaporación en un simple efecto. Cuando el vapor procedente de un evaporador se introduce como alimentación a la caja de vapor de un segundo evaporador, y el vapor procedente de éste se lleva después a otro, y después a un condensador, recibe el nombre de triple efecto. El método general de incrementar la evaporación por kilogramo de vapor vivo utilizando una serie de evaporadores entre la línea de vapor y el condensador recibe el nombre de evaporación de múltiple efecto [2].

Al estudiar la operación unitaria de levaporación, resulta indispensable el conocimiento de los valores que asumen las diferentes propiedades físicas y termodinámicas y el comportamiento de variables como la masa, entalpias y concentración durante el periodo en el que se evapora.

Las propiedades físicas y químicas de la solución que se está concentrando y del vapor que se separa tienen un efecto considerable sobre el tipo de evaporador que debe usarse y sobre la presión y la temperatura del proceso. Un sistema puede ser descrito en términos de un modelo simple que comprende balances de materia y entalpías asociados con los componentes del proceso; Generalmente, los evaporadores se representan por modelos matemáticos basados en balances de masa y energía.

Así el objetivo del presente trabajo es describir el comportamiento dinámico del proceso de evaporación de uno y múltiple efecto al vacío, para la construcción de un Laboratorio Virtual de Operaciones Unitarias a través del diseño y desarrollo de interfaces gráficas de usuario en un lenguaje de programación

\section{METODOLOGÍA}

\section{II.1 ELEMENTOS BÁSICOS}

La idea general sobre la construcción de un simulador de procesos que considere a la operación unitaria de evaporación, se basa en la disposición de equipos piloto de uno y doble efecto para la realización de prácticas experimentales con alto costo en materia prima y consumo de energía, En este sentido, el desarrollo de un laboratorio virtual permitira la reducción de costos de operación, y la posibilidad de realizar prácticas virtuales y la simulación de la operación en tiempo real para evaluar el desempeño del equipo ante diferentes condiciones de operación.

\section{II.2 BALANCES DE MATERIA Y ENERGÍA, Y PROPIEDADES}

Se realizo inicialmente una recopilación de datos de las propiedades físicas y termodinámicas necesarias para la construcción de modelos dinámicos que describan el comportamiento del fenómeno de evaporación en operación continua y por lotes. Estos conjuntos de datos fueron evaluados y se eligieron los que presentaron menor grado de error en un análisis de correlación. Se utilizó el programa Polymath 5.1, para realizar las correlaciones y el análisis de regresión de diferentes órdenes. Posteriormente, se codificaron estas funciones en un lenguaje de programación, para obtener los valores de las variables necesarias en los cálculos de la simulación del proceso.

Se desplegaron los balances de materia y energía correspondientes al proceso de evaporación en uno y en múltiple efecto, para el caso en estado estacionario y para el caso dinámico.

\section{II.3 INTERFACES GRÁFICAS DE USUARIO}

Las interfaces gráficas de usuario se realizaron en el programa QtCreator, se introdujo la imagen de un evaporador, se colocaron cajas de texto en donde el usuario introducirá los datos numéricos correspondientes a los datos, variables y propiedades termodinamicas necesarias para la simulación del proceso. Posteriormente, estas interfaces se codificaron y transformaron a extensiones que el lenguaje de programación Python pudiera reconocer, para el cálculo numérico y presentación de resultados.

\section{II.4 CÁLCULO NUMÉRICO}

Se procedió a la construcción del código fuente para la solución numérica y gráfica de los modelos matemáticos, correspondientes a los balances de materia y energía. Se utilizó el método de Runge-Kutta de cuarto orden para la solución de las ecuaciones diferenciales resultantes y las correlaciones mencionadas líneas arriba. El programa de cómputo se desarrolla de acuerdo al algoritmo mostrado en la Figura 1.

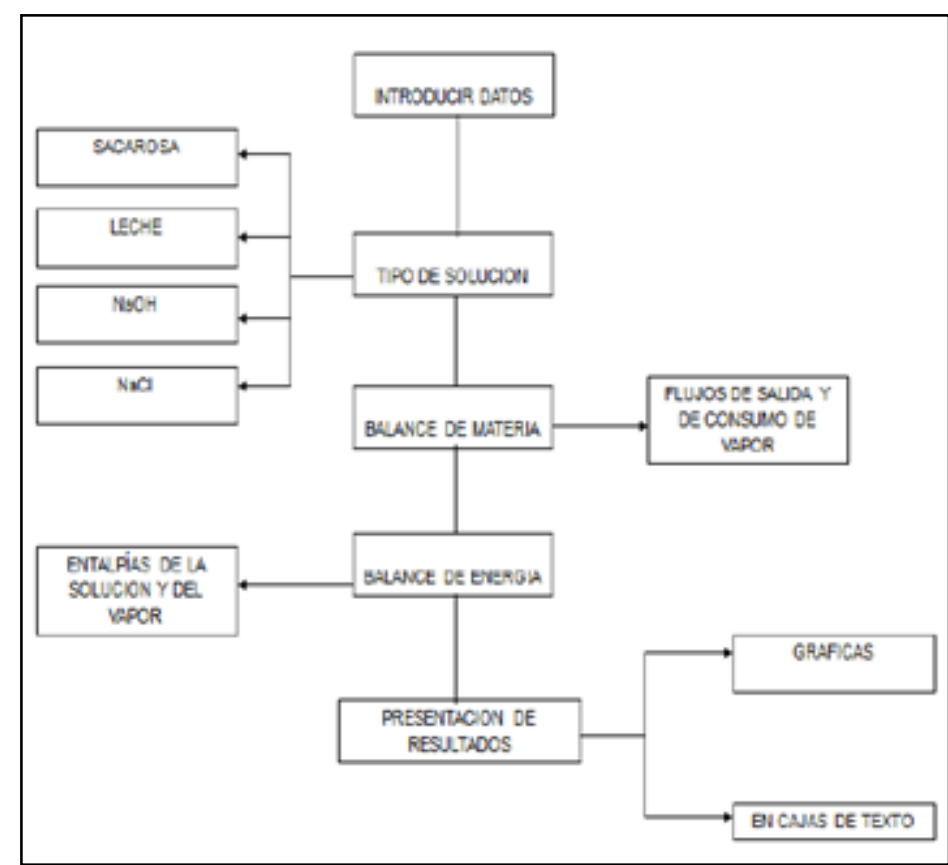

Figura1: Diagrama de flujo de la secuencnia de cáñculo.

Fuente: Los autores, (2018).

La codificación del algoritmo permite mostrar los resultados numéricos y gráficos en la interfaz de forma que el usuario puede observar los resultados numéricos, estacionarios o el comportamiento de las variables en el tiempo, según sea el caso, con la opción de guardarlos y abrirlos después con un formato de imagen.

El software desarrollado, describe el proceso de evaporación en estado estable, codificado en VisulaBasic, y en estado no estacionario, además presenta resultados en forma 
gráfica de las variables de proceso, tal como la masa retenida en el evaporador, la entalpía y la concentración, como funciones del tiempo.

\section{RESULTADOS}

La interfaz de operación, es la ventana principal desde donde se opera el programa. El usuario solo debe introducir los datos en las cajas de texto marcadas, para el caso de operación por lotes de simple efecto: concentración y temperatura del flujo de alimentación, presión del vapor saturado, área del evaporador, coeficiente de transferencia de calor y la concentración de salida deseada.

En el caso de operación continua, además se deben introducir características específicas como coeficientes de válvula y factores de geometría. Así mismo, para el inicio del cálculo númerico es necesario introducir las condiciones iniciales correspondientes a las variables de proceso representadas en las ecuaciones del modelo. El programa realiza el cálculo de las ecuaciones algebraicas necesarias para obtener los datos requeridos en la solución del modelo dinámico, y posteriormente resuelve el sistema de ecuaciones diferenciales por medio del método de Runge-Kutta.

Una vez que el programa termina el cálculo, sobre el esquema del evaporador aparecen los valores numéricos de las variables calculadas, como entalpías, presiones o temperaturas y el flujo de vapor de alimentación y salida. También, es posible observar el valor numérico de la elevación del punto de ebullición que se tenga a las condiciones de operación establecidas en la simulación. Los botones de la parte derecha, en el caso de simple efecto, o en la parte de abajo, para efecto múltiple, hacen que se activen las gráficas para el análisis e interpretación del comportamiento de las variables de proceso, tal como la entalpia, la masa, la concentración, en un caso, y además, en el otro caso, la altura del líquido dentro del evaporador.

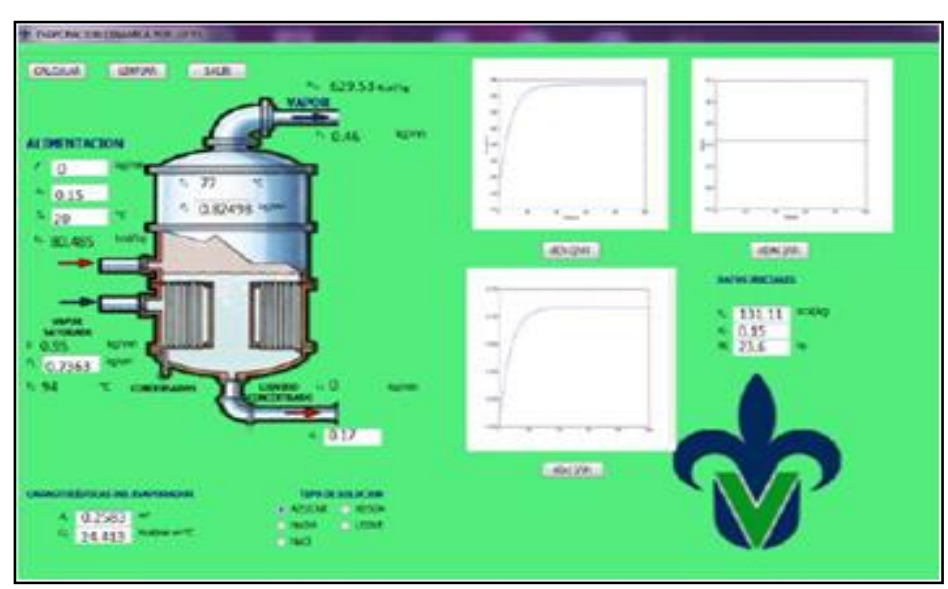

Figura 2: Interfaz gráfica de usuario de la evaporación de simple efecto en operación por lotes.

Fuente: Los autores, (2018).

Los resultados en forma gráfica de la simulación del evaporador de un solo efecto, para la operación por lotes, se presentan en forma ampliada en las Figuras 3, 4 y 5.

La entalpía de la solución a concentrar en el interior del evaporador, se muestra en la Figura 3. Mientras que, la variación de la masa del líquido en el evaporador, respecto al tiempo, se presenta en la Figura 4.

Además, en la Figura 5, se puede observar el comportamiento dinámico de la composición del líquido durante la operación del evaporador.
El análisis de los resultados gráficos que muestran los comportamientos dinámicos de las variables de proceso, permite observar y comentar que las variaciones son semejantes al comportamiento que se esperaria en forma experimental en el proceso real, bajo las condiciones establecidas en la simulación numérica.

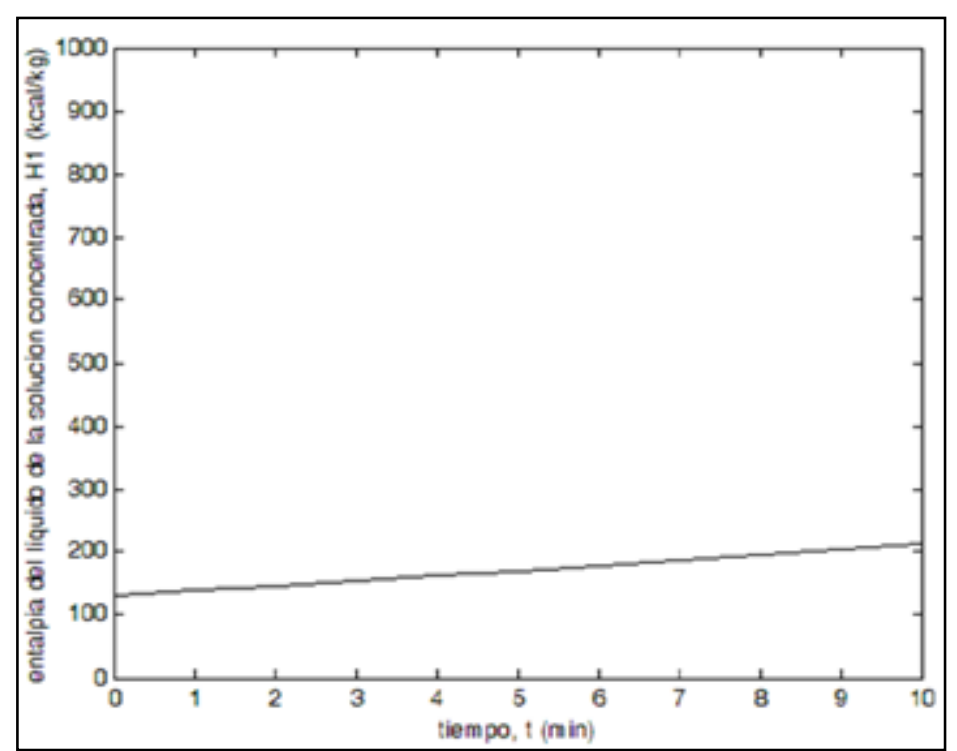

Figura 3: Gráfica entalpía del líquido en la solución contra el tiempo de procesamiento.

Fuente: Los autores, (2018).

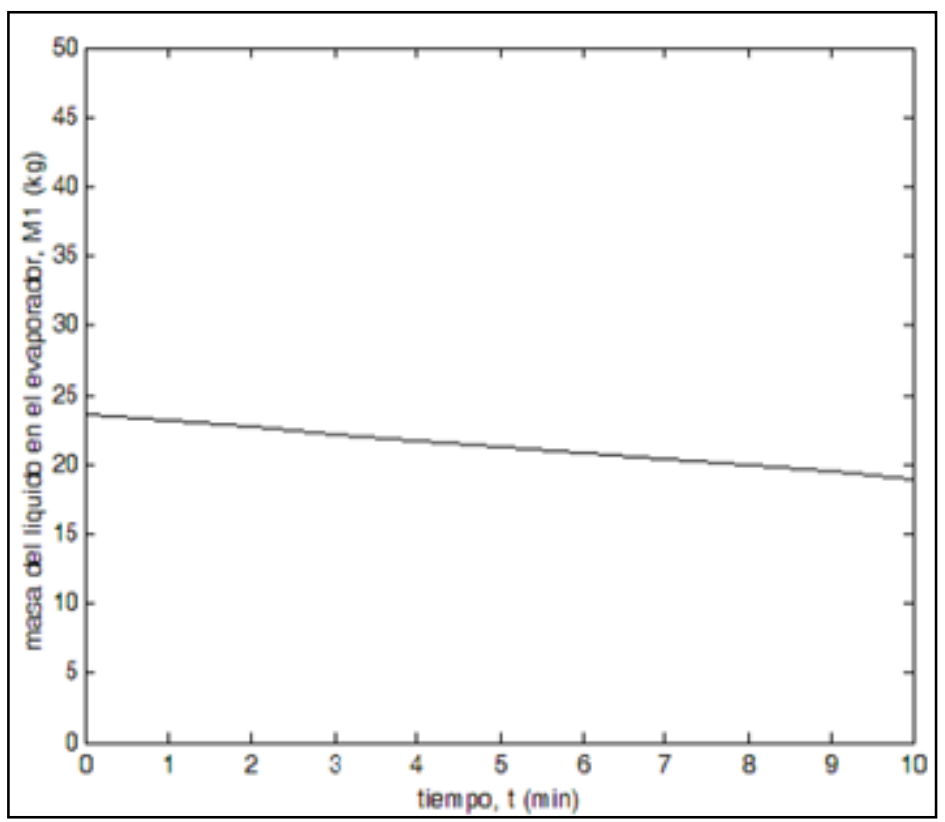

Figura 4: Gráfica masa de líquido en el evaporador contra tiempo de procesamiento.

Fuente: Los autores, (2018).

Para la operación en modo continuo, se utilizan las variables y parametros que se muestran en la Tabla 1 .

En el caso de la operación en mútiple efecto, la interfaz gráfica que se construyó para la presentación de los resultados numéricos se muestra en la Figura 6. 


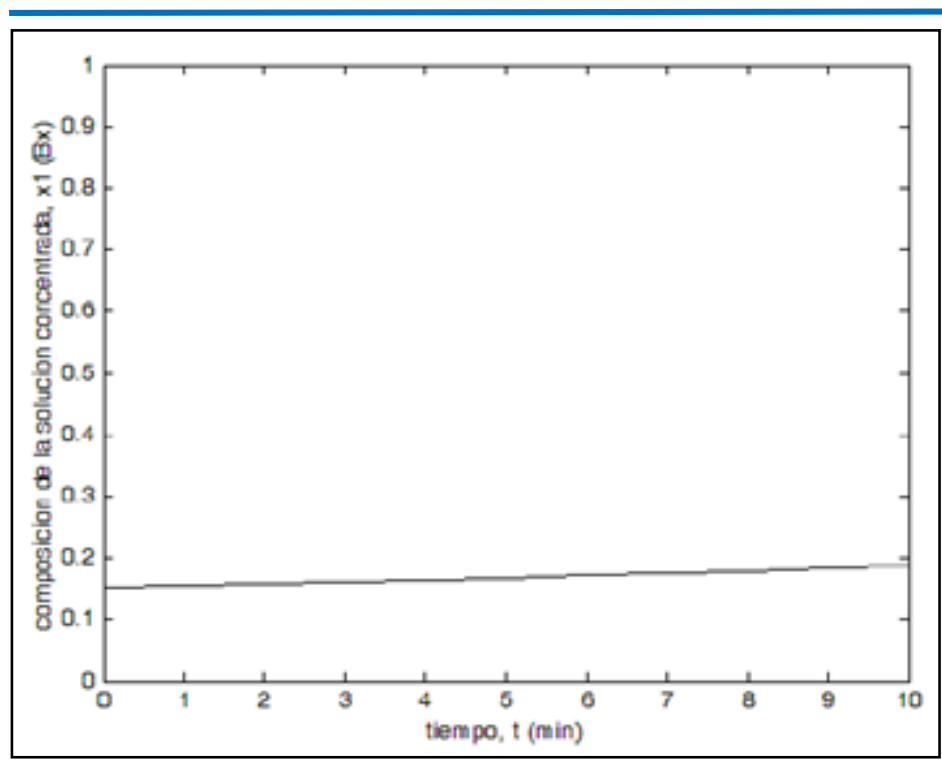

Figura 5: Gráfica composición del líquido en el evaporador contra tiempo de procesamiento.

Fuente: Los autores, (2018).

Tabla 1: Modo de operación continuo, variables y parámetros de proceso.

\begin{tabular}{|c|c|}
\hline $\mathrm{Pv}$ & Presión en la cámara del evaporador \\
\hline Pf & Presión en el fondo del tanque \\
\hline $\mathrm{Pe}$ & Presión del flujo de alimentación de solución diluida \\
\hline $\mathrm{Hv}$ & Entalpía de vapor \\
\hline At & Área del evaporador \\
\hline $\mathrm{Ah}$ & Área de transferencia de la chaqueta \\
\hline$\rho$ & Densidad del líquido \\
\hline$\rho \mathrm{v}$ & Densidad del vapor \\
\hline $\mathrm{U}$ & Coeficiente global de transferencia de calor \\
\hline Ts & Temperatura de condensación del vapor \\
\hline Tref & Temperatura de referencia \\
\hline $\mathrm{Cp}$ & Capacidad calorífica del líquido \\
\hline $\mathrm{Cv}$ & Coeficientes de válvulas \\
\hline $\mathrm{f}$ & Factor de geometría de la válvula \\
\hline ML & Masa retenida \\
\hline TL & Temperatura del líquido del evaporador \\
\hline $\mathrm{E}$ & Flujo volumétrico de alimentación al evaporador \\
\hline $\mathrm{Vv}$ & Flujo de evaporado que deja la fase líquida \\
\hline $\mathrm{V}$ & Flujo de vapor a la salida del tanque \\
\hline TG & Temperatura del líquido igual a la del vapor en la cámara \\
\hline
\end{tabular}

Fuente: Los autores, (2018).

Aquí se deben introducir los datos numericos necesarios, así como las condiciones iniciales para resolver el modelo dinámico del evaporador, en cada uno de los efectos se introducen

las condiciones iniciales. Se dispone de un sistema de coordenadas para cada uno de los efectos del múltiple. En cada eje de coordenadas se muestra el comportamiento de las tres variables que costituyen el modelo matemático de la evaporación. Adicionalmente, se dispone de la opción para mostrar una sola variable con una escala apropiada, suprimiendo las otras dos.

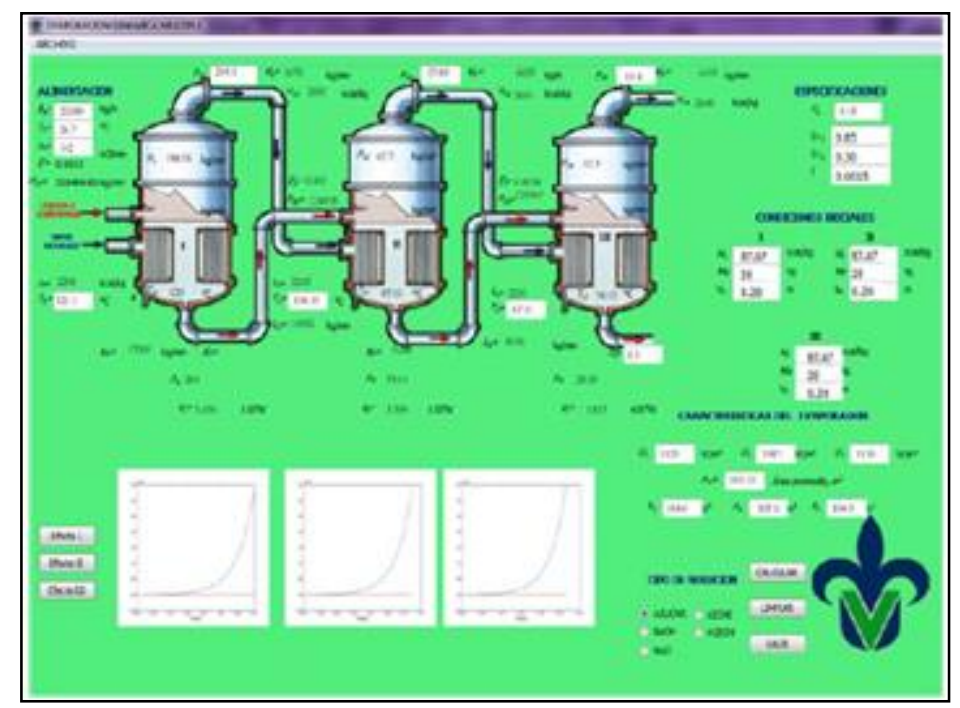

Figura 6: Interfaz gráfica para la simulación de un sistema de evaporación de triple efecto.

Fuente: Los autores, (2018).

\section{CONCLUSIONES}

Las interfaces graficas de usuario que se construyeron en la aplicación QtCreator permiten la descripción y análisis del comportamiento dinámico de las variables del proceso de evaporación para un solo efecto en operación por lotes y continúa. Además, cuenta con la opción de seleccionar diferentes tipos de soluciones a concentrar.

La codificación de los algoritmos de cálculo se realizo, en el caso de estado estacionario de un solo efecto en el lenguaje de programación Visual Basic, mientras que para el estado no estacionario, tanto en un solo efecto como en múltiple, los algoritmos se codificaron en el lenguaje de programación Python, debido a las características dinámicas que presenta, para la realización de gráficas y los métodos de solución de ecuaciones diferenciales integrados que posee.

El ambiente gráfico utilizado para la simulación del proceso de evaporación en uno y múltiple efecto representa un potencial didáctico para el análisis de esta operación unitaria, contribuyendo de esta forma al proceso enseñanza-aprendizaje en la currícula de Ingeniería Química.

\section{AGRADECIMIENTOS}

Deseamos agradecer a: Fondo Mixto (FOMIX) CONACYT-Gobierno del Estado de Veracruz por el soporte para el desarrollo de este trabajo, Proyecto: 109491.

\section{REFERENCIAS BIBLIOGRÁFICAS}

[1] McCabe, W.L., Smith J. C., and Harriott P., Operaciones Unitarias en Ingeniería Química. 6ed. México, McGraw Hill, 2002.

[2] Perry, R.H., Green D. W., Perry's. Chemical Engineers Handbook, 7ed. Nueva York: McGraw Hill, 1998.

[3] Geankoplis C. J., Procesos de Transporte y Operaciones Unitarias, 4ed. México: CECSA, 2003. 
[4] Ocon J., Tojo G. Problemas de Ingeniería Química. Tomo I. España: Aguilar, 1979.

[5] Holland C.D., Liapis, A. I., Computer Methods for Solving Dynamic Separation Problems. USA: Mc Graw Hill, 1983.

[6] Luyben W. L., Process Modelling Simulation and Control for Chemical Engineers, 2ed. USA: McGraw Hill, 1989.

[7] Stephanopoulus G., Chemical Process Control. USA: Prentice Hall Inc, 1984.

[8] Alonso P.A., López J., Salado M., Rocha J.A. Simulacion de Procesos en Ingeniería Química. México: Plaza y Valdes Editores, 2000.

[9]“Qt-Creator”. Octubre 2010; http://www.glatelier.org/2009/05/qt-creator-desarrollandoaplicaciones-rapidamente/ . 